\title{
A Catalog of Human Gut Microbial Sulfatases Reveals Structural Features Essential for Endobiotic Processing
}

\author{
S Ervin ${ }^{1}$ \\ ${ }^{1}$ University of North Carolina at Chapel Hill, Carrboro, NC \\ smervin@live.unc.edu
}

Human gut microbiome-encoded sulfatases are poised to reverse the action of mammalian sulfotransferases that inactivate endo- and xenobiotics as part of Phase II drug metabolism, impacting health and disease. In spite of their health implications, the numbers, types, and diversity of sulfatases within the human gut microbiome remain undefined. Here we present the first catalogue of sulfatase enzymes from the fecal metagenomic sequencing data within the Human Microbiome Project (HMP). Using protein structure-guided methods, we identified 728 distinct microbiome-encoded sulfatase proteins from the 4.8 million unique proteins present in the HMP Stool Sample database. We cloned, expressed, and purified a representative set of these microbial sulfatases and determined two novel crystal structures to pinpoint unique structural motifs essential for the processing of endobiotic-sulfate substrates. We show that gut microbial sulfatases differentially process hormone-sulfates in a manner dependent on active site architecture and quaternary structure. Finally, we demonstrate the inhibitors of human sulfatases have no effect on the microbial enzymes examined here. Taken together, data presented here provides unprecedented insights into the structural and functional diversity of sulfatases encoded by the human gut microbiome.

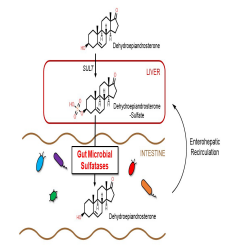

Figure 1

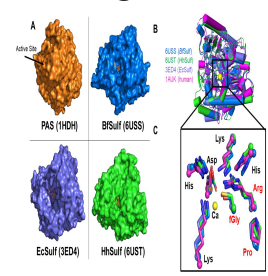

Figure 2

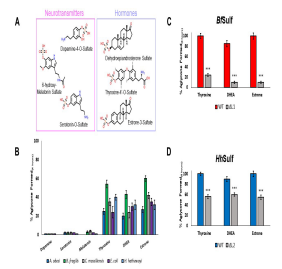

Figure 3

Acta Cryst. (2020). A76, a2 